\begin{tabular}{|l|l|}
\hline$I E-3$ & 21 \\
\hline
\end{tabular}

This paper describes a mathematical model for calculating and analyzing the effects of some factors on the economics of the existing automatic assembly systems and those might be developed in the future.

Results have been analyzed and compared with each other. Useful conclusions have been obtained and represent that the development of new grippers and feeders have very great benefit to the application of robots in the assembly systems. The conclusions of this work have good agreement with an experimental case study made to assemble a 24 -piece gear box.

\title{
INTRODUCTION
}

In this paper a technique has been developed to evaluate and analyze the cost of the assembly process carried-out by an automatic assembly system. The use of mechanized and automated devices to replace manual assembly operations is known as Automatic Assembly. An automatic assembly machine usually consists of a transfer system, automatic workheads vacant work stations, and inspection stations. When considering an assembly of a product, a manufacturer has to take into account many factors that affect the choice of assembly system; the most important factor is the cost of assembly.

One of the major attributes of this technique is the ability to analyze the cost of separate determination of the detailed factors involved; including parts quality level, capital cost of equipment including overheads, the assembly operator rate, number of shifts, number of assemblies, downtime, cycle time, number of operators equivalent to a piece of equipment... etc The effects of the change in product design and product style variations have been, also, studied in this work.

*

Lecturer at Prod.Eng. Dept., Alex.Univ., Egypt, and working for Kuwait Institute of Technology. 


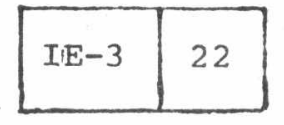

$r$

An important application of programmable equipment of the automatic assembly is the application of robots (programmable workheads) to multisation indexing machines. These mechines are basically dedicated machines, where the single task workheads are replaced by robots capable of performing more than one assembly task.This scheme provides flexibility in that the machine can be designed to match more closely the output volume required. In addition, variation of product style can be accomodated since the workheads are under computer control and can be commanded to select parts among alternatives available at a particular station, $[1,2]$.

In the following study, the mathematical model will be applied only on the assembly indexing machines.

\section{NOMENCLATURE AND ASSUMED VALUES}

$C \quad=$ Assembly cost per completed assembly

$\mathrm{C}_{\mathrm{c}}=$ Cost of work carrier per work station (1000\$)

$\mathrm{C}_{\mathrm{f}}=$ Cost of automatic feeding device $(5000 \$)$

$\mathrm{Cg}_{\mathrm{g}}=$ Cost of gripper per part $(500 \$)$

$\mathrm{C}_{i}=$ Cost of a piece of capital equipment

$\mathrm{C}_{\mathrm{m}}=$ Cost of hand-1oaded magazine ( $\left.500 \$\right)$

$c_{\text {pf }}=$ Cost of a programmable feeder (from eqn.23 or 5000\$)

$\mathrm{C}_{\mathrm{pg}}=$ Cost of a programmable gripper (from eqn.22 or 10000\$)

$C_{r}=$ Cost of robot (from eqn. 10)

$\mathrm{C}_{\mathrm{sg}}=$ Cost of a special gripper

$c_{t}=$ Cost of transfer device per work station (10000\$)

$\mathrm{C}_{\mathrm{w}}=$ Cost of work head $(1000 \$)$

$\mathrm{D} \quad=$ Number of changes in product design

$\mathrm{d}=$ Ratio of defective parts to accepted parts (0.01)

$f \quad=$ Number of degrees of freedom

$\mathrm{K}=$ Equivalent factor, capital cost per operator per year per shift $(2500 \$)$

$\mathrm{Np}=$ Number of product changes during machine life

$\mathrm{N}_{\mathbf{s}}=$ Variations of product style

$\mathrm{n} \quad=$ Number of parts to be assembled

$\mathrm{p} \quad=$ Proportion of defective parts will cause machine stcoppage $(1.0)$

$\mathrm{q}=$ Proportion of parts which a gripper change is required $(0.5)$

$\mathrm{R}=$ Operator's rate (15000 \$/year)

$\mathrm{S} \quad$ Number of shifts (3)

$\mathrm{T}=$ Assembly time per acceptable assembly

$\mathrm{t}=$ Cycle time, time for each assembly task

$t_{\mathrm{g}}=$ Gripper change time ( $5 \mathrm{sec}$. )

$t_{r}=$ Operator time to restart machine ( 30 sec.)

$\mathrm{y}=$ Machine life ( 10 years) 
In practice some of the defective parts would pass through the feeding devices and automatic workheads but would not be assembled correctly and would result in the production of an unacceptable assembly. Therefore, the total assembly time, to produce $\mathrm{N}$ assemblies, including acceptable and unacceptable assemblies, is given by $\mathrm{Nt}+$ pNnt $\mathrm{d}$ where the acceptable assemblies will be $N-(1-p) N n d$. In this case the average assembly time of acceptable assemblies is given by :

$$
T=\left(N t+p N n t_{r} d\right) /(N-(1-p) N n d)
$$

In the case of all defective parts will produce stoppage of the machine, i.e $p=1$, the assembly time per part is given by

$$
T=t+n t_{r} d
$$

The total operating cost of the machine per unit time deals with the acceptable and unacceptable assemblies and includes cost of operators and overheads, actual operating costs and depreciation rate. The unacceptable assemblies, (1-p) Nnd, need an extra operator time te to dismantle the assembly and replace the nondefective parts back in the appropriate feeding devices. Therefore, the total operating cost per unit time is given by [1i]

$$
c_{T}=c_{o p}+(1-p) n d \cdot t_{e} R /\left(t+p n t_{r} d\right) \ldots . . \cdot
$$

The operating rate of the machine, $C_{\text {op,is }}$ equal to the annual rate for the equivalent number of operators ( $n_{0}$ ) to replace an equipment per shift, then

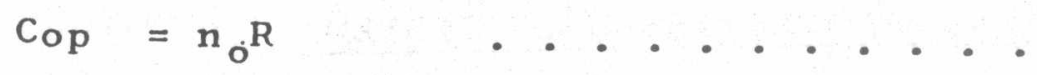

Therefore, the cost of a piece of capital equipment, $c_{i}$, has a proportion relation with the annual wage rate of the equivaIent operators during the actual useful life of the equipment (y years.). Thus :

$$
c_{i} \propto n_{0} S y R
$$

or

$$
\mathrm{n}_{\mathrm{O}} \mathrm{R}=\mathrm{c}_{\mathrm{i}} \mathrm{R} / \mathrm{SyK} \mathrm{K} \quad \text {. . . . . . . }
$$

where, $k$ is an equivalent factor and represents by the cost of capital equipment used to replace one operator per shift per year.

Usually for many factories the cost of the equivalent operators to replace a piece of equipment $\left(n_{0} R\right)$ is ranged between 0.3 and 1 times of the cost of capital equipment $\left(C_{i}\right)$. Therefore, the value of factor $K$ can be calculated according to the other items.

The operating cost per unit time for a piece of equipment can be obtained from the above relation as:- 


\begin{tabular}{|l|l|}
\hline$I E-3$ & 24 \\
\hline
\end{tabular}

SECOND A.M.E. CONFERENCE

$$
C_{o p}=C_{i} R / S y K \quad \ldots \ldots \ldots
$$

Hence, the total rate for the machine and operators will be given, from equations 3 and 6 as :

$$
\left.C_{T}=R\left[3+\left(C_{i} / S y K\right)+(1-p) n t e^{d /(t+p n t}{ }_{r} d\right)\right] \ldots .
$$

where the total rate for all operators engaged on the machine represented by $3 R, i . e \quad 300 \%$ overheads.

Generally the assembly costs per assembly are given by

$$
\mathrm{c}=\mathrm{T} \mathrm{C}_{\mathrm{T}}
$$

\section{AUTOMATIC ASSEMBLY SYSTEM}

In the case of automatic assembly system the cost $C_{i}$ may be represented as the summation of the costs of the system equipment, such as, transfer device $\left(C_{t}\right)$, work carrier $\left(C_{c}\right)$, feeder $\left(C_{f}\right)$, and workhead $\left(C_{w}\right)$.

Thus

$$
c_{i}=n\left(c_{t}+c_{c}+C_{f}+C_{w}\right) \quad . \quad . \quad . \quad .
$$

and the assembly time $T$ is given from equation (1)

Using equations 7,8 and 9 the assembly cost may be calculated and represented as functions of number of parts $(n)$ and production rate(assembly frequency, 1/T). Figure (1) shows these relations according to the values assumed before [ 3$]$

\section{PROGRAMMABLE ASSEMBLY SYSTEM}

One of the applications of programmable equipment to automatic assembly is the application of robots (programmable workheads) to multistation indexing machines.

The cost of these robots will be assumed according to the practices, [1] as

$$
\mathrm{C}_{\mathrm{r}}=25000+8000 \mathrm{f} \$ . \cdot \ldots(10)
$$

where $f$ is the available number of degrees of freedom.

In this case, and if $n_{i}$ parts are assembled at each station, it is assumed that, due to lower production rate, hand-ioaded magazines and gripper for each robot are used instead of parts feeder, then, $C_{i}$ of eqn ( 8 ) become,

$$
c_{i}=\left(n / n_{i}\right) \cdot\left(C_{r}+c_{t}+c_{c}\right)+n\left(c_{g}+c_{m}\right) \cdot \cdot
$$

and the assembly time is given by

$$
T=\left(n_{i} t+p n t_{r} d\right) /(1-(1-p) n d) \ldots . .
$$

the assembly cost of this case is represnted in fig.(2). 


\section{FACTORS AFFECTING THE ECONOMIC ANALYSIS}

The programmability feature of the robots can be used to accomodate small change in product design, without changing the design of the workhead.

Assuming the number of changes in product design during the life of the machine is $D$ and eachproduct design change involves only one part requiring one new feeder and gripper. Then $c_{i}$ of eqn. (8) wi11 be

$$
c_{i}=\left(n / n_{i}\right)\left(c_{r}+c_{t}+c_{c}\right)+(D+n)\left(c_{g}+c_{f}\right) \ldots .
$$

and assembly time is given by eqn(13).

Using the programmable workheads (robots) it is easy to assemble the variant product styles. Number of styles ( $\left.N_{s}\right)$ is known as the ratio of the number of parts available at each station $\left(\mathrm{nN}_{\mathrm{S}}\right)$ to the number of parts which are used to assemble one product $(n)$, then the equations for the indexing machines with robots will be

$$
c_{i}=\left(n / n_{i}\right)\left(c_{r}+c_{t}+c_{c}\right)+n_{s}\left(c_{g}+c_{f}\right) \ldots . .
$$

and the assembly time is given by equation (12)

Similarly,in the case of automatic assembly, without robots, the effect of change in product design and variation of product style are represented respectively as

$$
\begin{aligned}
& c_{i}=n\left(C_{t}+C_{c}\right)+(D+n)\left(C_{w}+C_{f}\right) \\
& c_{i}=n_{s}\left(C_{t}+C_{c}+C_{w}+c_{f}\right)
\end{aligned}
$$

and the assembly time as in eqn. (1)

\section{ASSEMBLY CENTERS USING ROBOTS}

In the assembly centers one assembly robot with one or two arms is used. These types of robots need a program to perform complicated series manipulations, and a special gripper that will handle all the parts in an assembly. Denoting the cost of such a gripper by $C_{s g}$, the cost $C_{i}$ will be

$$
\mathrm{C}_{i}=\mathrm{C}_{\mathrm{r}}+\mathrm{C}_{\mathrm{c}}+\mathrm{C}_{\mathrm{sg}}+\mathrm{n} \mathrm{C}_{\mathrm{m}} \cdot \ldots .
$$

The cost, using the ordinary gripper, is represented by

$$
c_{i}=c_{r}+c_{c}+n\left(c_{g}+c_{m}\right)
$$

Before performing an assembly operation the robot must replace the previously used ordinary gripper in the rack and select the new gripper. Therefore, the mean assembly time, considering gripper change time, is given by

$$
T=n\left(t+q t_{g}+t_{r} d\right) . . .
$$

For the case where no griper changes, $q=0$, and the asembly time of the robots using a special gripper is given by 


$$
\mathrm{T}=\mathrm{n}\left(\mathrm{t}+\mathrm{t}_{\mathrm{r}} \mathrm{d}\right)
$$

From equations 18 and 19, the values of the assembly cost/part can be calculated to represent that q has no effect on the cost with change of the production rate, as shown in Fig.( 3 ). This figure shows, also, that the assembly cost increases with increase of q value at certain number of parts to be assembled
$(\mathrm{n})$.

The economic cost of a special gripper can be obtained by equating the assembly costs of cases of using ordinary and $18)$, then the economise costs represented by equations $(8,17$,

$$
\mathrm{C}_{\mathrm{Sg}}=n \mathrm{C}_{\mathrm{g}}+\left(\mathrm{q} \mathrm{tg}_{\mathrm{g}} /\left(\mathrm{t}+\mathrm{t}_{\mathrm{r}} \mathrm{d}\right)\right)\left[3 \mathrm{~S} y \mathrm{~K}+\mathrm{C}_{\mathrm{r}}+\mathrm{C}_{\mathrm{C}}+\mathrm{n}\left(\mathrm{C}_{\mathrm{g}}+\mathrm{C}_{\mathrm{m}}\right)\right]
$$

Considering the possibility of product changes, a programmable gripper should be used. The cost of this gripper should be affected by the number of different products to be assembled during the life of the machine $\left(N_{p}\right)$ and the cost of the special gripper for each product, then the economic cost of the
programmable gripper is given by:

$$
\mathrm{C}_{\mathrm{pg}}=\mathrm{N}_{\mathrm{p}} \mathrm{C}_{\mathrm{sg}}
$$

Assuming $Z$ percentage of parts should be presented by a programmable feeder in the assembly center, and other parts are will be

$$
\mathrm{C}_{\text {feeder }}=(\text { TRn/SyK })\left(Z C_{p f}+(1-Z) N_{p} C_{f}\right)
$$

The economic cost of the programmable feeder is obtained, by differentiation of the above relation and equating by zero, as:

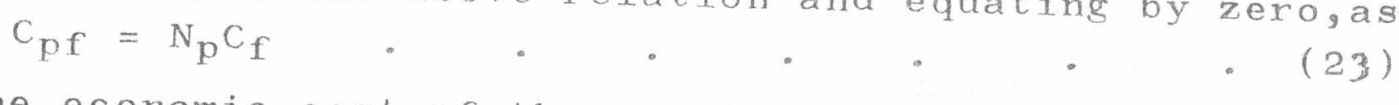

where the economic cost of the special feeder $C_{f}$ is given by oquating the operator loading cost for one part and the cost time per parti is $t_{1}$. Therefore,

$$
C_{f}=t_{1} \text { SyK/n } N_{p}\left(t+t_{r} d\right) \text {. }
$$

A reduction in the me an assembly time can be achieved by using a robot with two arms, one arm is selecting the next Thus the mean assembly time will be and the assembly cost is given by

$$
\mathrm{T}=\mathrm{n}\left(0.5 t+t_{r} \mathrm{~d}\right) . \ldots .
$$

$$
\mathrm{C}=\mathrm{TR}\left[3+(1 / \mathrm{SyK})\left(2 \mathrm{C}_{r}+\mathrm{NP}_{\mathrm{P}}\left(\mathrm{C}_{\mathrm{C}}+\mathrm{nC}_{\mathrm{g}}+\left(\mathrm{nN}_{\mathrm{S}}+\mathrm{D}\right) \mathrm{C}_{\mathrm{m}}\right)\right)\right]
$$

A predicted assembly center, may be known as the complete and feeder that will handle, where the programmable gripper The assembly cost of such systemisible parts, might be used. 


\begin{tabular}{|l|l|}
\hline$I E-3$ & 27 \\
\hline
\end{tabular}

$$
C=\operatorname{TR}\left[3+(1 / \mathrm{SyK})\left(2 \mathrm{C}_{\mathbf{r}}+\mathrm{N}_{\mathrm{p}} \mathrm{C}_{\mathrm{c}}+2 \mathrm{C}_{\mathbf{p g}}+\mathrm{nN}_{\mathbf{S}} \mathrm{C}_{\mathrm{pf}}\right)\right] .
$$

The robot arms may be had only three degrees of freedom, and over heads ratio is only $200 \%$. i.e., equal to $2 \mathrm{R}$. It is clear that this proposed system, if developed, would be capable to handle all possible parts.

To represent the capabilitites of this proposed system and other existing systems, a comparison will be made for the same requirements.Therefore, equations of this systems will be given as following:

- for automatic assembly system:

$$
\begin{aligned}
C_{i} & =n N_{s}\left(C_{t}+N_{p} C_{c}\right)+N_{p}\left(D+n N_{s}\right)\left(C_{w}+C_{f}\right) \\
\text { and, } T & =t+n t_{r} d \ldots \text { for } p=1
\end{aligned}
$$

- for programmable assembly systems

$$
C_{i}=\left(n / n_{i}\right)\left(C_{r}+N_{s}\left(C_{t}+N_{p} C_{c}\right)\right)+\left(n N_{s}+D\right) N_{p}\left(C_{g}+C_{f}\right) \ldots
$$

and $T=n\left(t+q t_{g}+t_{r} d\right)$

- for aseembly centers with 2-arms robot

$$
C=\operatorname{TR}\left[3+(1 / \text { Sy.K })\left(2 C_{r}+N_{p}\left(C_{c}+n C_{g}+\left(n^{n}+D\right) C_{m}\right)\right)\right] . .
$$

and, $T=n\left(0.5 t+q t_{g}+t_{r} d\right)$

- for complete programmable assembly centers

$$
\begin{gathered}
C=\operatorname{TR}\left[2+(1 / \operatorname{SyK})\left(2 C_{r}+N_{p} C_{c}+2 C_{p g}+n N_{s} C_{p f}\right)\right], \cdots \\
T=n\left(0.5 t+t_{r} d\right),
\end{gathered}
$$

and $\mathrm{C}_{\mathrm{pg}}$ and $\mathrm{C}_{\mathrm{pf}}$ is given by equs. (22 \& 23) respectively.

The values of all items are assumed as shown before.

Figures 4 through 7 show some relations of these equations.

\section{EXPERIMENTAL CASE STUDY}

Specifically to dispel some of the reservations about robots and in general to consider new manufacturing possibilities, groups at RCA Laboratories (Princeton, NJ,U.S.A), [4], are investigating various phases of flexible manufacturing. The initial task was to consider the feasibility of robotically assembling a 24-piece gear box subassembly. Designing a robotic gripper to handle all these varied parts would be very difficult. And, employing many different grippers would only slow the process. Thus an attempt was made to modify the product (reduction in some parts and elimination of some parts) so that it would be easier to assemble. Of course, no reduction in product capability or quality could be accepted.

A Hirata AR-300 robot was proposed because of its repeatability and low cost. This type of robot, called SCARA (selective compliance assembly robotic arm), and consists of a 4-degrees of freedom one-arm robot, controlier, and pendant. 

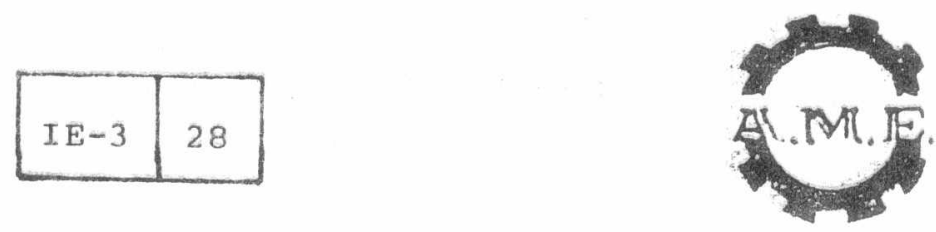

SECOND A.M.E. CONFERENCE

$6-8$ Hay 1986 , cairo

$r$

The handling, orienting, and feeding of parts, as they arrived from vendors, are formidable jobs. In general, all parts required reorienting for the robot. If the parts are relatively small and not delicate, a vibratory bowl feeder can be used. Therefore, 5 vibratory bowl and two trays are used in this system.

In a short time, the system was upto rate, and including the short downtime for replacing emptied pallets, it averaged 16.5 secands per assembly.

In this syotem, the greatest effort and costs were in part feeding, not in programming or using the robot. Part feeding curtails flexibility, increases costs, greatly increases floor space requirements, and lengthens concept-to-delivery time: Further, part feeding equipment accounted for almost $70 \%$ of the purchased part costs for the system. In the future, the use of robots for assembly will depend on improvement in methods to deliver oriented parts to the robotic area and also in gripping methods. As automation becomes more relality and robots play a greater role, competition will only become more aggressive, requiring a stepped-up search for and implementation of new and innovative techniques in all future engineeing and manufacuring projects. Therefore, according to the system classification of this paper this case study will be classified as assembly center with one-arm robot. Figure? represents the actual relation of this case study with respect to other systems at 24 parts, as number of parts to be assembled.

\section{DISCUSSION AND CONCLUSION}

1. A mathematical model is developed to analyse the assembly cost, using an automatic assembly system. This model has some factors affecting the economical analysis. The applications of this model are carried out, in this work, for some assembly
indexing systems.

2. For case of automatic assembly system, the assembly cost per part increases with increase the assembly cycle time, as shown in Fig. (1), and has minimum cost at certain number of parts. This figure shows that the assembly cost per part decreases with increase the production rate. Also, increase of number of parts decreases the cost.

3. The assembly cost per part, in case of programmable assembly systems, has a critical number of parts at which the lowest cost is obtained by using one of the two assembly systems, as shown in Fig. (2). Figure (2) shows, also, that an station ( $\left.n_{i}\right)$ decreases the cost.

4. Figure (3) shows that no effect of gripper change on the production rate. At the same time any increase of $q$ or $n$
increases the cost. 
5. From equations 28 through 31 it is clear that an increase of each of $\mathrm{D}, \mathrm{N}_{\mathrm{S}}$ or $\mathrm{N}_{\mathrm{p}}$ increases the cost for any of existing assembly systems. These relations are shown in figure (4) for the case of 2 -arms robot assembly center, equation ( 30$)$.

6. The assembly cost of the complete programmable assembly center,with economical values of the programmable gripper $\left(\mathrm{C}_{\mathrm{pg}}\right)$ and feeder $\left(\mathrm{C}_{\mathrm{pf}}\right)$ (eqns. 22 and 23$)$, is represnted with two conditions in Fig. (5). The same cost is represented for an assumed values, $C_{p g}=10000 \$$ and $C_{p f}=5000 \$$, and gives the lowest assembly cost per part, but as a prediction of future.

7. In figures 6 and 7 , curves are marked as: a-automatic assembly systems (eqn.28); b-programmable assembly systems (eqn.29), c-assembly centers with 2-arms robot (eqn.30), dcomplete programmable assembly centers with economical values of $\mathrm{C}_{\mathrm{pg}}$ and $\mathrm{C}_{\mathrm{pf}}$, and e-complete programmable assembly centers with assumed values of $C_{p g a n d} C_{p f}$. These figures show that the lowest assembly cost per part, at all, is achieved by the complete programmable assembly centers, if developed, at any condition.

8. The assembly operation of the experimental work is considered as case c, assembly centers with one-arm robot. The experimental assembly cost per part is very closed to the corresponding theoretical case, as shown in Fig.7, while has about 6.75 times of the assembly cost using the proposed system, case d.

9. Finally, in the futute, the use of robots for assembly will be depend on an improvement in methods to deliver oriented parts to the robotic work area. The development of the required programmable grippers and feeders and the design of products for assembly would extend immensely the areas of application of programmable assembly automation. The proposed assembly centers would change the assembly method completely, and would be most economical to assemble different products.

\section{REFERENCES}

1. Boothroyd, G.,et.al., "Automatic Assembly", Dekker Press, U.S.A, 1982 .

2. Groover,M.P., "Automation, Production Systems and CAM", Prentice-Hall, U.S.A. 1980 .

3. Dewhurst,P.,et.a1., "CAD for Assembly-A Microcomputer System", Conf.of NUMRC, U.S.A. 1982.

4. Jahn Aceti, "Robotic Gearbox Assembly", Mfg.Eng., SME, USA, Apri1, 1985 . 


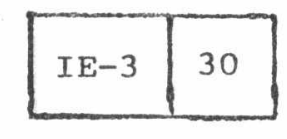

$r$
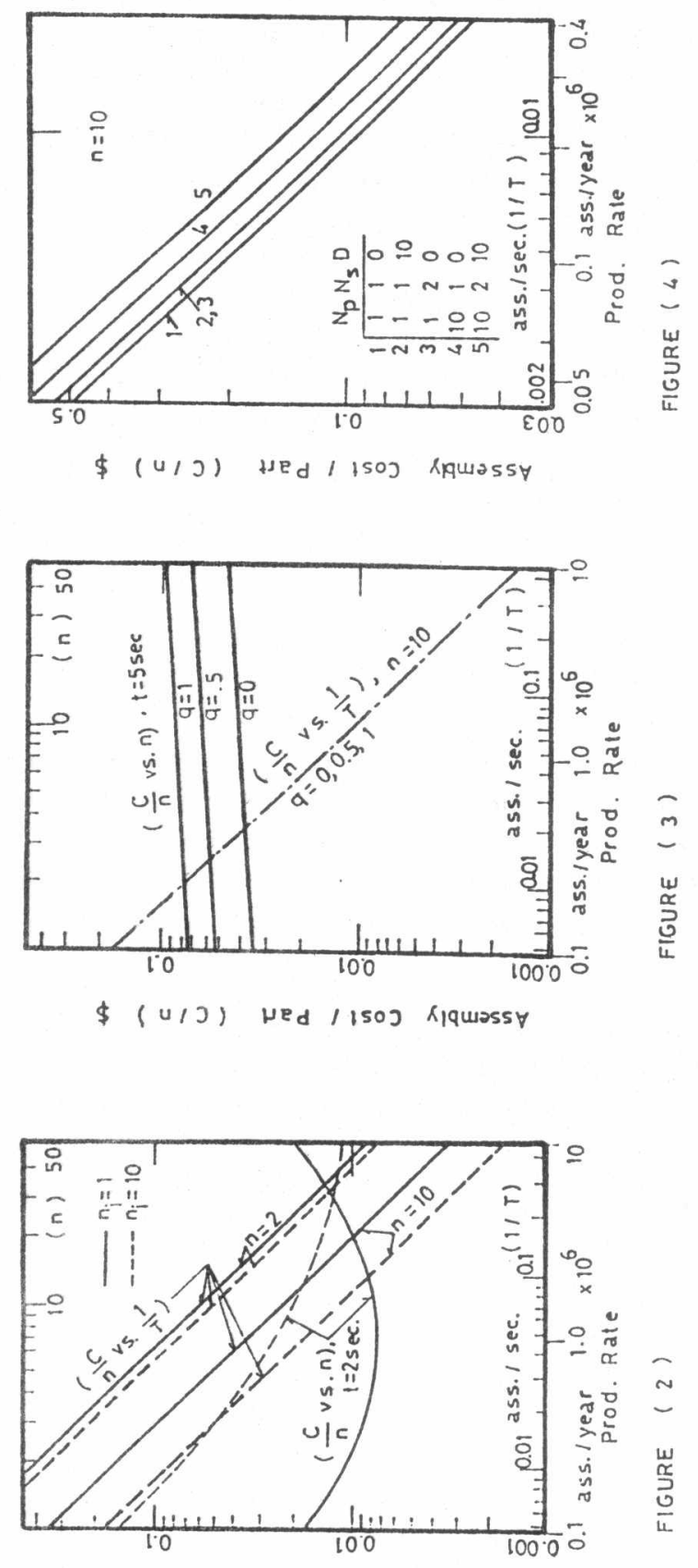

$\$(4 / J)$ liedl isog klquass
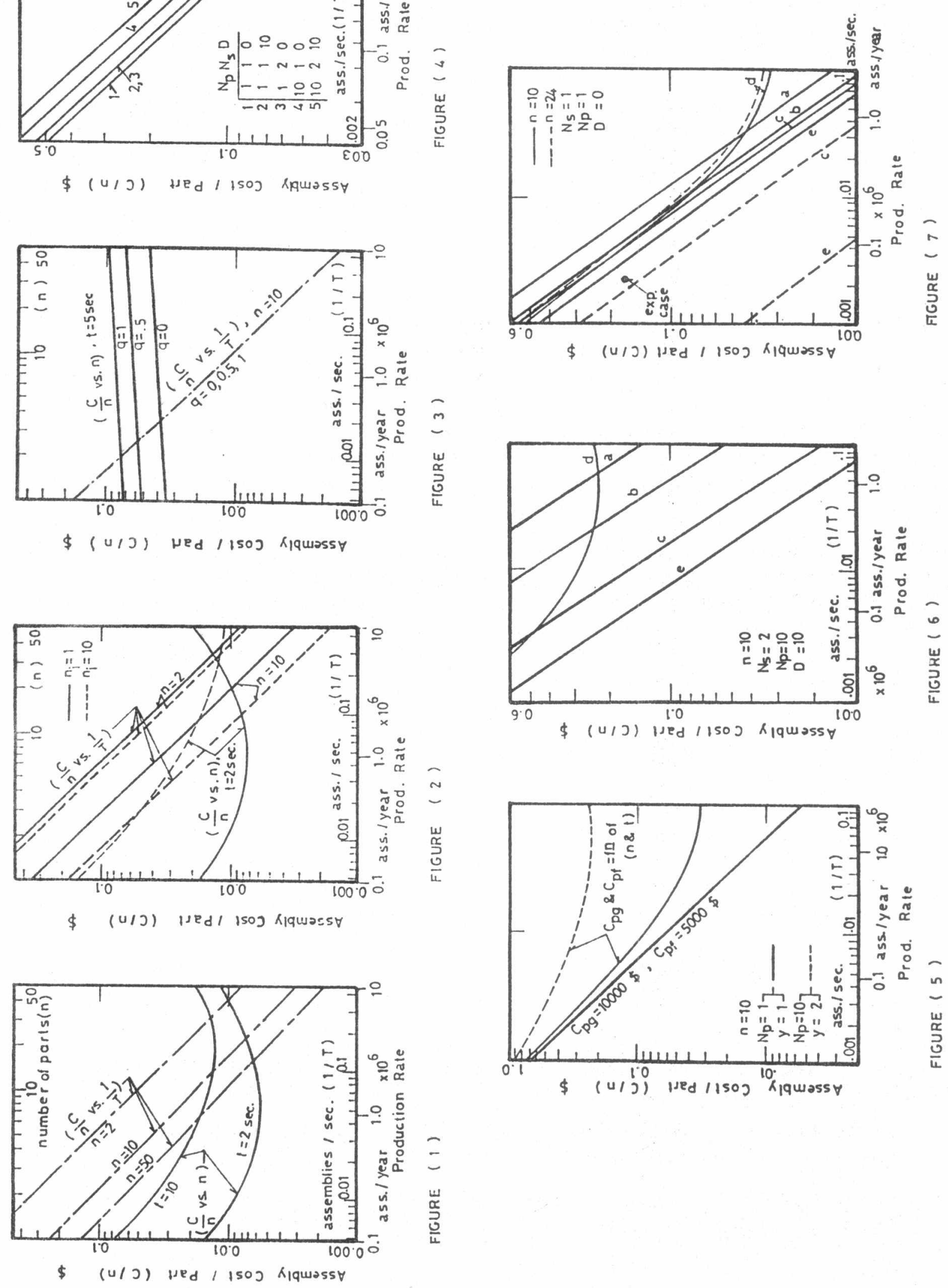